\title{
L'appropriation des nouvelles technologies de la mobilité par le tourisme : nouveaux enjeux créatifs
}

Johan Vincent

\section{OpenEdition}

1 Journals

Édition électronique

URL : http://journals.openedition.org/tourisme/380

DOI : 10.4000/tourisme.380

ISSN : 2492-7503

Éditeur

Éditions touristiques européennes

\section{Édition imprimée}

Date de publication : 1 décembre 2014

Pagination : 62-74

ISSN : 2109-5671

\section{Référence électronique}

Johan Vincent, "L'appropriation des nouvelles technologies de la mobilité par le tourisme : nouveaux enjeux créatifs », Mondes du Tourisme [En ligne], 10 | 2014, mis en ligne le 30 septembre 2015, consulté le 10 décembre 2020. URL : http://journals.openedition.org/tourisme/380 ; DOI : https:// doi.org/10.4000/tourisme.380

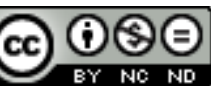

Mondes du tourisme est mis à disposition selon les termes de la licence Creative Commons Attribution - Pas d'Utilisation Commerciale - Pas de Modification 4.0 International. 


\title{
L'appropriation des nouvelles technologies de la mobilité par le tourisme :
} nouveaux enjeux créatifs

\author{
JOHAN VINCENT \\ Chercheur associé au Cerhio (UMR 6258) \\ [johan.vincent@gmail.com]
}

Résumé. La créativité n'est pas le seul fait des technologies et des technophiles : les acteurs veulent s'adapter à ce/ceux qu'ils considèrent comme de "nouveaux tourismes" ou de "nouveaux touristes", liés à l'innovation numérique. Pour l'instant, l'offre reste verticale : les professionnels sont maîtres des processus qu'ils mettent à la disposition des publics, perçus essentiellement comme des consommateurs. La culture de création par des touristes sur des produits tiers paraît encore trop balbutiante pour s'épanouir. Associer le public au processus créatif, c'est prendre des risques vis-à-vis du message de confiance, c'est aussi des investissements parfois conséquents, des retombées mal cernées. A contrario, c'est acquérir des avantages pour la diffusion du tourisme et de l'innovation sur l'ensemble d'un territoire, et c'est être réellement partie prenante de l'inévitable jeu concurrentiel avec les infomédiaires. Nouvelles technologies et tourisme créatif sont une nouvelle porte d'accès au territoire qu'il n'est pas facile psychologiquement de franchir.

Abstract. Creativity is not reserved to technology and technophiles: the actors of tourism want to adapt their practices to a "new tourism" or to "new tourists", in connection with technological innovation. For the moment, the offer is vertical: the professionals still control the processes they make available to the tourists (regarded as consumers). The culture of creation from tourists on third party products still seems too stammering to bloom. Involving the public into the creative process means taking risks (message of confidence, investments, results). But it also means taking advantages in matter of tourism diffusion and of innovation, and being a stakeholder of the inevitable competition with the infomediaries. New technology and creative tourism are a new step which is not, psychologically, easy to take. 
e tourisme est confronté depuis quelques années à un champ neuf qui s'ouvre à lui : le m-tourisme (ou tourisme de la mobilité), peu de temps après avoir dû répondre aux enjeux de l'e-tourisme (ou tourisme lié à internet). Le rapport Prospective du m-tourisme, paru en novembre 2011, parle d'ailleurs d'une double révolution à ce sujet (Idate et Kanopée, 201 I). Les smartphones et les tablettes, dont le volume de ventes a dépassé celui des PC en 2011, sont devenus les nouveaux outils pour promouvoir les territoires, créer de l'intérêt et renouveler la curiosité. L'innovation numérique s'inscrit dans le redéploiement de l'offre touristique : nouvelles politiques d'accueil des publics, de médiation, de communication, d'expositions, de documentation, d'édition, de diffusion culturelle et artistique, de partenariat et de recherche de mécénat. Le mtourisme oblige à réfléchir aux usages qui émergent, et implique, souvent localement, une créativité pour les exploiter, les développer, voire les inventer. Il s'inscrit aussi dans un mouvement général dont il est le contemporain : le tourisme créatif, expression inventée en 1993, surtout depuis les années 2000, et le pullulement des initiatives à ce sujet (Richards, 20I I, pp. 1237-1238). L'idée des industries créatives, dont le tourisme est l'un des pans, est de rompre avec l'état secondarisé de la culture et du patrimoine pour s'en servir afin de stimuler l'initiative créatrice et l'investissement privés (Toussaint, 2012, p. 63) : réinventer les mises en valeur et les expériences touristiques, par l'intermédiaire des professionnels et/ou des touristes, pour générer un phénomène immersif : une nouvelle façon de vivre, dans sa création ou à travers sa création.

Lors de la deuxième conférence internationale sur le tourisme créatif : "Le tourisme à l'heure du touriste créatif”, les 6 et 7 décembre 2012, les professionnels ont démontré que la créativité n'est pas le seul fait des technologies et des technophiles ; les usages ont également une grande importance. Éric Seulliet (2012), président du think tank La Fabrique du futur et intervenant à ce colloque, associe trois mots-clés pour parvenir à une réussite des projets : co-création, lead users (qu'on peut rapprocher des gate-keepers) et communauté d'usagers. Les acteurs doivent innover parce qu'ils sont confrontés à ce qu'ils considèrent comme de "nouveaux" touristes. Comme les nouvelles technologies sont facteurs de créativité, ils explorent des possibilités jusqu'alors inconnues : l'exploitation de la géolocalisation est évidemment caractéristique. Ils cherchent aussi à valoriser les savoirfaire des visiteurs - visiteurs déjà protéiformes, comme les a analysés Jean-Didier Urbain dans L'Idiot du voyage (2002) et Le voyage était presque parfait (2008). Cette belle volonté ne cache-t-elle pas des enjeux sous-jacents, qui confinent tourisme créatif et nouvelles technologies à un état de superficialité ? Il convient de reconnaître que, pour l'instant, les études se cantonnent aux relations $B$ to $B$ (business to business) et que le contentement du consumer, le touriste, reste pour ainsi dire inconnu, faute d'études scientifiques qualitatives. Le propos de cet article se fixera, lui aussi, du côté de l'offre.

L'article propose de faire un état des lieux des nouveaux outils $(\mathrm{QR}$ codes, guides touristiques dématérialisés, applications muséales, applications ludiques...) et de leurs contenus, afin de cerner comment les commanditaires et les concepteurs créent de l'intérêt à partir d'une ressource dématérialisée. Le choix d'une forme de communication n'implique pas les mêmes réponses. Avec certaines applications, le touriste dispose d'un outil pour préparer (avant), profiter (pendant) et promouvoir son voyage (après). Avec la réalité augmentée, il bénéficie dans sa poche d'une table panoramique d'où il peut accéder à des contenus. Avec le QR code (code barres bidimensionnel), il jouit d'un accès à des contenus mais à partir d'un affichage à un endroit précis ou sur un prospectus. Les acteurs du tourisme doivent donc adapter leurs discours et innover : le fontils ? D'un autre côté, le touriste estil si différent avec l'arrivée de la technologie ? Une réflexion sur l'environnement général dans lequel les créations peuvent se fixer est nécessaire, même si elle sera modeste faute d'études réellement fournies sur ces relations.

Finalement, à la lumière de ces dynamiques qui peuvent apparaitre contradictoires, l'article pose la question d'une créativité exacerbée nécessaire chez les acteurs du tourisme. 
Elle est pour l'instant balbutiante : chacun reconnaît que nous sommes, potentiellement, à l'orée d'une nouvelle vision touristique, ce que confirment tous les intervenants de la conférence du 2 décembre 2011 sur les nouveaux usages des technologies de l'information et de la communication. Il faut aussi avoir conscience qu'en 2012 moins de $4 \%$ des offices de tourisme en France ont une application Iphone dédiée (Guidéapolis, 20।2). Le rôle d'animateur et d'accompagnateur (conseil) se trouve renforcé, à plusieurs échelles, pour faire connaître et faire vivre les créations - l'aspect relatif aux réseaux, toujours sous forme de solutions liées au tourisme de mobilité, voire éventuellement aux marques (marketing territorial), entre en ligne de compte, comme on peut le voir pour d'autres structures (musées, par exemple). Plus largement émerge la limite à la créativité, que nous interrogerons dans cette partie plus prospectiviste. Le secteur touristique dispose de beaucoup d'atouts, mais il est régi par de nombreuses craintes. L'article repose essentiellement sur des exemples français.

\section{NOUVELLES TECHNOLOGIES, NOUVEAUX TOURISMES ?}

Au-delà des pratiques, il faut analyser comment la technologie se matérialise sur le terrain. Trois formes se distinguent aujourd'hui en matière de tourisme : le circuit touristique ou patrimonial dématérialisé ; la réalité augmentée ; l'application smartphone.
Un tourisme de circuit : QR codes

Sans facilitation d'accès à l'information, il était impossible qu'un circuit touristique puisse être créé à partir de la seule technologie. Or le code barres bidimensionnel, qu'il suffit de "flasher" (certains parlent de "flashcode" pour désigner ce type de code barres) pour lancer l'ouverture d'un texte, d'un sonore ou d'une vidéo, facilite cet accès. Le code barres QR a été créé par l'entreprise japonaise Denso Wave, en 1994, qui l'a diffusé sous licence libre en 1999. À la fin des années 2000, il est devenu l'un des codes barres bidimensionnels les plus populaires dans le monde.

Si les premiers usages des codes barres bidimensionnels ont d'abord été rares, à cause du faible équipement des touristes et surtout d'une grande méconnaissance de cet outil, ils sont désormais une des entrées préférées pour un circuit d'interprétation du patrimoine. La première commune à avoir commencé à véritablement exploiter les capacités du smartphone semble être Savins (Seine-et-Marne) : elle a associé à des codes barres bidimensionnels un service exploitant la vidéo. Faute de financements suffisants pour embaucher un guide permanent, la commune a opté en avril 2009 pour ce procédé technologique qui permet de visiter l'église, fermée au public en dehors des offices religieux, et le lavoir de l'Étain. Si la vidéo sur l'église est un récit érudit sur fond musical, la vidéo sur le lavoir de l'Étain s'appuie sur le témoignage de l'ancien maire, Martial Goutte, qui raconte la remise en état du lavoir et les pratiques qui s'y déroulaient il y a un peu plus d'un demi-siècle (images et reconstitutions actuelles) $)^{(1)}$. Dans la station balnéaire de Barbâtre (Vendée), le circuit Balis'Âges a été spécifiquement élaboré en 2010 pour associer contenu touristique patrimonial à partir de témoignages et technologie des QR codes. Sont disponibles un petit texte de présentation de l'endroit, des vidéos, des images et des informations complémentaires $^{(2)}$. La philosophie du programme ne repose pas sur une érudition mais sur les sentiments de la population envers son territoire : filiation, attachement, fierté. Dans les deux cas, l'opération s'appuie sur le potentiel local : le touriste est accueilli par l'habitant, qui partage ses connaissances et ses passions. Dans le même temps, des circuits patrimoniaux dématérialisés plus standardisés, avec texte, images et parfois vidéos scénarisées, ont été mis en place en France : Blaye, Bordeaux, Houplin-Ancoisne, Tournan-en-Brie... $8000 Q R$ codes sont annoncés en Seine-et-Marne. Les touristes sont donc invités à bouger sur tout le territoire, $\mathrm{y}$ compris dans les communes rurales jusqu'alors délaissées faute de budget à consacrer à la mise en valeur du patrimoine.

À l'instar des circuits d'interprétation classiques, les circuits fonctionnant avec des $Q R$ codes ont l'avantage de fonctionner à tout moment de l'année et à tout moment de la journée, dès que le réseau téléphonique fonctionne. Le visiteur n'a 
pas besoin d'aller chercher le matériel puisqu'il dispose déjà de son smartphone (pas de limitation liée à l'ouverture d'un organisme comme l'office du tourisme ou la mairie). Il n'est pas restreint par la disponibilité des témoins, puisque ceux-ci sont enregistrés et racontent leurs histoires sans usure. Il peut donc s'organiser pour suivre les différentes étapes du circuit ou pratiquer un tourisme opportuniste, au hasard de ses promenades. Ce tourisme se veut sans contraintes et, bien que le visiteur soit invité, par une communication dédiée, à réaliser un parcours, c'est un "tourisme de cueillette". Ainsi, les statistiques révèlent que, malgré la basse saison hivernale, le circuit Balis’Âges est fréquenté. Sur les six premiers mois d'exploitation (du 2 octobre 2010 au 11 avril 2011), les différentes étapes ont été consultées par une cinquantaine de personnes individuelles (entre 34 et 91 , selon les étapes), avec un nombre de visites compris entre 50 et 121 . Trois ans plus tard, la fréquentation reste importante, malgré une petite érosion ${ }^{(3)}$. Les statistiques révèlent notamment le croisement des circuits touristiques puisque les points de Balis’Âges les plus fréquentés sont ceux qui sont situés sur le circuit pédestre et cyclable faisant le tour de l'île de Noirmoutier. C'est un usage à la carte, désormais plus facile, que le touriste commence à privilégier : un tourisme de l'entre-soi, qui se développe autour du lien entre les hôtes (les habitants et leurs “secrets") et les visiteurs. Pour l'instant, ces circuits innovants n'ont pas dépassé les limites du circuit d'interprétation "en dur", qu'on ne peut changer après sa pose, au contraire des circuits fonctionnant avec des $Q R$ codes. Le touriste est cantonné dans un rôle de consommateur, sans qu'il puisse proposer du contenu ou ne serait-ce qu'interagir.

\section{Un tourisme de vision : réalité augmentée}

La réalité augmentée permet d'approcher une dimension importante pour le touriste : l'immersion. Afin de ne plus ressembler à "l'idiot du voyage", le touriste se sert de la technologie, malgré un aspect encore expérimental. Pour l'instant, les produits sont essentiellement fixés au smartphone: se superpose en temps réel, grâce à la fonction de l'appareil photo, une image virtuelle sur les éléments de la réalité. Ils permettent une hypertextualisation de la réalité. Cet aspect technologique, plus complexe à réaliser, est l'un des derniers à avoir été adoptés par les offices de tourisme.

Dans un premier temps, la médiation a été essentiellement documentaire. L'application Cultureclic est l'une des plus anciennes : elle délivre une somme d'informations sur les lieux culturels se situant dans un périmètre proche. Puis la dimension ludique a été promue. Jessica Fèvresde Bideran (2013, p. 16) remarque d'ailleurs que ces applications, comme Cluny-vision ou Jumièges $3 \mathrm{D}$, sont classées dans la catégorie "voyage" d'Itunes, quand Cultureclic apparaît dans la catégorie "enseignement”. La réalité augmentée doit désormais révéler la face cachée des choses, voire les faire réapparaître. Le château fort de Cherbourg, pourtant disparu, est aujourd'hui visible. Le parcours pédestre en réalité augmentée "Imayana", mis en place depuis le 15 septembre 2012 par l'office de tourisme de Bordeaux, propose une découverte du cœur historique au XVIII siècle avec du matériel dédié. Les formes narratives tiennent compte des actions du visiteur autour de neuf "points d'expérience" (Plantier et Barbier, 20I3, p. 30). Le processus de création d'un parcours en réalité augmentée est relativement lourd : il faut maîtriser le contenu de ce qui va être montré, concevoir un logiciel adapté à la modélisation de ce contenu, anticiper la mise en scène de l'ensemble, ce qui implique des va-et-vient entre les différentes étapes du projet (Cieutat, 20 I3, p. 52 et p. 54-55).

L'un des avantages de la technologie repose sur l'accompagnement toujours possible de la mise en valeur du site. Le marché technologique s'ingénue maintenant à développer des éléments plus discrets que le port du smartphone : les lunettes connectées, les montres connectées..., selon le processus de miniaturisation que le téléphone portable a connu dans les années 1990. La réalité augmentée risque d'être durablement la porte d'accès à une multitude d'informations, remplaçant progressivement la carte ou la liste, les deux modes préférentiels actuels. Après l'informatique ubiquitaire, nous aurions l'information ubiquitaire, dans sa vision totale. Ce n'est pas sans poser 
quelques défis, telles l'absence de gêne du regard ou l'exploration des lieux sans surcharge mentale, comme le relèvent Nehla Ghouaiel, JeanMarc Cieutat et Jean-Pierre Jessel (20I I). De plus, jusque dans quel degré de réalité ou d'irréel le touriste veut-il se mouvoir ? La réalité augmentée ne doit pas se confondre avec la réalité virtuelle. Pour l'instant, la distinction entre les deux univers : exister dans le monde réel pour la première, exister dans un monde autre que celui où le corps se trouve pour la seconde (Cieutat, 20 I3, pp. II 12), n'est pas clairement établie dans les produits créatifs touristiques. Le processus créatif en matière de réalité augmentée, du fait de sa complexité, restera durablement aux mains des professionnels.

\section{Un tourisme de participation : applications smartphone}

L'application smartphone limite l'information à son objet. La plupart des guides touristiques (Routard, Gallimard, Hachette, Petit Futé, Lonely Planet, Michelin...) ont une présence par des sites ou applications mobiles, qui ne couvrent cependant pas l'ensemble de leurs collections. Il s'agit souvent, pour ces éditeurs, d'applications "vitrines" aiguillant vers un service payant. Les comparateurs et sites d'avis spécialisés sur le tourisme sont en revanche peu présents sur ces médias, si l'on excepte les majors tels que TripAdvisor, Holidaycheck, Easyvoyage ou Jetcost. Le développement de sites internet mobiles optimisés est systématique chez les spécialistes du tourisme, alors qu'il est occasionnel pour les géants du net (Idate et Kanopée, 20 I I, p. 98). Pour les acteurs institutionnels du tourisme, l'application permet la création d'une attraction spécifique, tout en conservant une maitrise forte, et d'ailleurs bien souvent totale, des contenus. Certaines structures sont d'ailleurs explicitement éditrices de leur application. Le comité régional du tourisme du Limousin lance ainsi son application Tèrra Aventura, proposant près d'une centaine d'aventures, des décryptages d'énigmes et un univers numérique peuplé de Poï'z et de BadPoï'z, qui fonctionne après inscription. L'agence de développement et de réservation touristique de l'Aisne a lancé SaintQuentin Tourisme, Laon Tourisme et Soissons Tourisme. Le comité départemental du Tarn propose deux applications : l'une sur les circuits touristiques, l'autre sur un jeu d'enquête interactif sous forme de quiz, à chaque fois sur le Tarn.

Les premières applications pour les organismes locaux d'information et de promotion datent de 2010. Un recensement effectué mi-2011 fait état de 180 applications pour smartphones et 65 sites web mobiles, dont 29 ont été développés conjointement avec les applications. Cet inventaire est loin d'être exhaustif concernant ces derniers, car il n'existe pas d'annuaires de sites mobiles (Idate et Kanopée, 201 I, p. I 10). Lors de son étude du marché des applications des offices de tourisme en 2012, limitée aux produits disponibles sur la plate-forme d'applications d'Apple,
Guidéapolis dénombre 130 applications pour Iphone et 7 applications pour Ipad. Le retour de satisfaction des touristes paraît correct. Le guide touristique Mobil'iti de la Vallée de Clisson, inaugurée en juillet 2009 sur Iphone, téléchargé par un peu plus de 5000 personnes en six mois, a globalement satisfait le public, qui l'a perçu comme une innovation enrichissant la visite effectuée à son rythme (Arbey et al,, 20 I 0, pp. 42-46).

L'application n'est pas nécessairement l'étape la plus complexe à réaliser pour une structure touristique. Les auteurs du mémoire sur la stratégie future de l'office de tourisme Médoc-Océan rappellent que le plus difficile, c'est inciter le visiteur à télécharger l'application (Arbey et al., 20 I 0, p. 42). Ensuite, la structure touristique peut engager avec le mobinaute une relation plus proche, grâce à une actualisation automatique. Elle peut également l'avertir d'une nouveauté ou d'un évènement par des notifications dites en mode push, qui sont des messages d'alerte prenant la forme d'un petit message en haut de l'écran d'accueil du téléphone. La communication de masse n'est toutefois pas sans risque, en particulier celui d'importuner son interlocuteur, ce qui implique une réflexion dans la mise en place d'une telle stratégie. Cette interrogation est révélatrice du problème du positionnement des applications : alors qu'elles pourraient être un lieu de création collective, entre les institutionnels et les visiteurs, elles tendent pour l'instant à se substituer aux objectifs d'un site internet mobile, 
où la communication est essentiellement verticale.

\section{NOUVELLES TECHNOLOGIES, NOUVEAUX TOURISTES ?}

Les nouvelles technologies de la mobilité n'interviennent pas sur des populations sans savoir-faire. Elles répondent à certaines de leurs attentes ou accompagnent des évolutions sociales touristiques.

\section{La géolocalisation potentielle- ment partout}

L'un des atouts des technologies de la mobilité repose évidemment sur la géolocalisation. Elle permet un renseignement immédiat du touriste, sans qu'il ait à annoncer sa position. Ce gain de temps peut être consacré à d'autres occupations, trouvées dans une application smartphone ou sur un site internet. La technologie doit permettre de combattre un mal que l'on connait souvent dans les files d'attente des parcs d'attraction et des musées, que les professionnels, quand ils le font, tentent de combattre pour l'instant en adoptant un affichage mural informatif complémentaire à l'attraction. La contrepartie de cette facilité, c'est l'impatience du touriste face à des moments d'attente qu'il ne peut occuper ou un sentiment de fastidiosité quand il doit remplir un document pour accéder à des données. La géolocalisation s'inscrit également dans une perspective originale de découverte : le geocaching, dont le but est de rechercher un objet ou un lieu sur un territoire. Le syndicat d'initiative de Villedieu-les-Poêles loue ainsi des GPS depuis 2012 : il propose aux touristes la visite de lieux insolites après la résolution d'une énigme. Deux temps forts au printemps (geocaching jeune public "Mais où se cache Clochette" et rallye GPS expert) ont été proposés afin d'éveiller la curiosité du public. D'autres territoires créent des applications smartphone ad hoc pour renouveler l'intérêt des populations pour le territoire, en lui permettant de le redécouvrir sous un autre angle, plus ou moins fictionnel. Grâce à l'application "Portées disparues", l'office de tourisme de Poitiers propose ainsi de partir à la recherche de personnages féminins disparus à Poitiers au cours des siècles. Employée de cette manière, la technologie est le nouveau relais de croissance touristique, après les chemins de randonnée des années 1990 et les pistes cyclables des années 2000 .

Grâce à une diffusion plus large des technologies mobiles, la distinction entre espace de plein air et espace fermé tend à s'estomper. Le touriste comme cible est dorénavant atteignable presque partout, à l'exception des zones blanches du réseau téléphonique. En effet, la géolocalisation n'a été longtemps possible que dans les espaces où le smartphone pouvait recevoir le signal satellitaire : les murs épais stoppent ce signal. La technologie permet dorénavant une géolocalisation dans les bâtiments, à partir d'un réseau de bornes wi-fi disposées tous les 20 à 30 mètres. La précision de la position de l'utilisateur est de 3 à
5 mètres. Deux sociétés françaises sont d'ailleurs citées comme des entreprises en pointe en la matière (Insiteo et Pole Star). Depuis février 2012, ces systèmes fonctionnent dans les centres commerciaux La Maquinista à Barcelone et Les Quatre Temps à la Défense, uniquement sur les téléphones Android car la société Apple n'ouvre pas, actuellement, sa puce aux développeurs. Insiteo souhaite combiner wi-fi et signaux GPS pour atteindre une précision d'un mètre (Amiot, 20|2). Cette technologie implique un investissement dans un équipement supplémentaire, là où l'utilisation du signal satellitaire s'apparente plutôt à la position du passager clandestin. Néanmoins, les plus grosses structures trouveront intérêt à s'équiper, surtout si elles parviennent à fédérer plusieurs usages autour de cette possibilité : usage marchand (promotions), usage pratique ou informatif (contenus des audio- et vidéo-guides en cloud), usage ludique (geocaching), sans surcoûts pour les populations étrangères si c'est le wi-fi qui est exploité. Le m-tourisme peut renforcer les collaborations entre des acteurs aux objectifs différents.

\section{L'indépendance des pratiques}

À partir des années 1990, d'autres agents que les acteurs institutionnels commencent à être reconnus comme interlocuteurs officiels par les touristes, au-delà de la communication informelle que le visiteur peut obtenir de ses hôtes ou des autres visiteurs. Cette évolution se traduit, par exemple, par le phénomène des gree- 
ters. Signifiant en anglais "hôtes" ou "comité d'accueil", les greeters sont présentés comme des clés ouvrant chacune une porte donnant sur un aspect différent du territoire. Ils se développent dans le monde à l'échelle des villes. Lynn Brooks, à l'initiative du mouvement en 1992 , constatant que New York intimidait les gens, a imaginé une forme d'accueil touristique basée sur la rencontre avec des habitants, pour permettre aux visiteurs de comprendre la ville et ses occupants. Le mouvement est en forte progression : en octobre 2010, 27 greeters sont recensés dans le monde, dont 9 en France ; en septembre 2013, 52 dans le monde et 20 en France ${ }^{(4)}$. En France, ils sont gérés par des comités départementaux du tourisme, des offices de tourisme ou des associations loi 1901. Le tourisme devient participatif. Dans leur dossier de presse, les Greeters de Nantes, association regroupant une trentaine de personnes-ressources, font témoigner Hervé, 38 ans : "C'est comme si on faisait la visite avec un copain que l'on n'a pas vu depuis un moment. C'est une belle aventure humaine à vivre $^{(5)}$." Les visites sont proposées sur mesure, même s'il se dégage des thématiques, pour découvrir les adresses typiques ou atypiques, les trésors cachés, les rendez-vous, les petites habitudes et les grandes histoires de la région. C'est une autre forme du tourisme de l'entre-soi.

Sans que cela soit une règle intangible, les touristes prennent donc progressivement l'habitude de gérer leur visite. Les audioguides ont libéré le cheminement des visiteurs dans les musées, qui peuvent les parcourir de façon totalement autonome (Deshayes, 2002, pp. 25-26). Ils sont perçus comme une alternative à la visite guidée, parfois vécue comme disqualifiante car positionnant le visiteur dans une logique d'assisté. À l'usage, c'est le rapport à l'espace qui induit un parcours de visite. Les outils technologiques sont promus comme une manière d'accéder à cette indépendance, même si, dans les faits, elle est limitée. La transposition sans adaptation, dans les premières applications, du contenu de guides papier ou de sites internet a été un échec patent. Les éditeurs de guides se sont rendu compte que la formule papier et la formule électronique sont en fait complémentaires : tant que le wi-fi gratuit n'est pas plus répandu, le numérique est un complément de voyage en amont, pour la préparation (Bostnavaron, 2012). Néanmoins, certains produits, essentiellement des applications smartphone, sont déjà partiellement modelables. Ils encouragent la participation du public, via les réseaux sociaux ou, plus timidement, en modifiant des aspects de l'outil. Des guides touristiques dématérialisés, comme, dans ses dernières versions, l'application Mobitour développée par l'entreprise Phonlabs, proposent de donner son avis sur les sites visités, de prendre des photos destinées à compléter ou à remplacer les premiers visuels, après vérification. Ces fonctions, rares pour l'instant, sont loin d'être la norme. Et, de toute façon, la culture de création sur des produits tiers de la part des touristes paraît encore trop balbutiante pour s'y épanouir. Lors d'une discussion impromptue avec Didier Simon, directeur de l'office de tourisme de la baie de Saint-Brieuc, celui-ci révèle que le public interagit peu. Le touriste créatif n'est pas la figure principale du touriste pour l'instant.

\section{L'assimilation des informations et leur réutilisation}

En théorie, les populations touristiques sont appelées à s'approprier les outils de cette indépendance. L'analyse des comportements touristiques des digital natives, ceux qui sont nés avec la technologie, sera particulièrement intéressante à mener. Les services mobiles distribués par le smartphone ou la tablette sont pour l'instant vécus sous un aspect pratique : ils permettent la planification du voyage et sont choisis parce qu'ils facilitent les prises de décision sans être confronté à une saturation d'informations. Leurs usages n'ont pas provoqué encore de rupture par rapport aux pratiques habituelles du touriste. Or le mélange des motivations dans la découverte des destinations est une caractéristique forte chez la génération $\mathrm{Y}$ (Pendergast, 2010, p. 13-14). Pour s'en convaincre, il suffit de prendre conscience du succès des visas vacances-travail dans plusieurs pays, comme l'Australie ou le Canada. Le touriste n'est donc plus aujourd'hui systématiquement seulement un touriste. La personnalisation du service, progressivement proposée, est particulièrement appréciée. Wang, Park et Fesenmaier (201 I, 
p. 9) qualifient les applications personnalisables de "perfect concierge" ("concierge parfait"). Après la mise à disposition de l'information, encouragée par internet, débute donc la domestication de cette information. C'est une réflexion appliquée depuis le printemps 2012 par plusieurs moteurs de recherche pour internet. Dorénavant, l'information doit être directement utile à l'usager, qui ne doit pas avoir à la chercher et à la manipuler. Le choix de l'application a plus d'importance pour le mobinaute que la portée quantitative du marché d'applications lui-même, quand celui-ci a atteint une taille critique.

Cette évolution implique une appropriation anticipée de l'intérêt touristique : le touriste ne doit plus seulement être informé, il doit être mis en situation, un peu comme un acteur qui lit une pièce théâtrale ou un scénario cinématographique. Il doit pouvoir dépasser le stade de l'observateur, dans lequel il est généralement cantonné, par le biais des commentaires comme seul mode d'expression. Les nouvelles technologies ont donc aussi pour objectif de permettre la projection. Cela n'empêche pas que d'autres touristes restent spectateurs de ces manifestations : ils sont touristes de la mise en tourisme d'un territoire. Cette double présence apparaît déjà dans les reconstitutions grandeur nature où, au cours d'une ou plusieurs journées, certains jouent une vie quotidienne médiévale ou la reconstitution de batailles. La rencontre de personnages charismatiques, devenus pierre angulaire d'un musée, donne à la journée une forme événementielle. Pour Claude Origet du Cluzeau et Patrick Vicériat (2009, p. 155), "par leur caractère éphémère, [ces types d'évènement] parviennent souvent à suivre, précéder et, apparemment, orchestrer la frénésie de changement et la demande de convivialité $d u$ public”. Là où la participation reste le fait d'initiés, l'utilisation des nouvelles technologies peut rompre les barrières - même si elles peuvent aussi apparaître comme des barrières. D’un autre côté, la nouveauté des comportements créatifs permet de comprendre la prudence des acteurs institutionnels en la matière. Sans y être hostiles, les structures touristiques optent pour une position ambivalente face à ces innovations sociales aux conséquences multiples.

\section{UNE CRÉATIVITÉ EXACERBÉE ?}

L'ensemble des acteurs du secteur touristique a un rôle à jouer pour encourager et bonifier le tourisme créatif, car la technologie seule ne suffit pas.

\section{Le renouvellement de la confiance}

Créer ne suffit pas. Il faut accompagner l'assimilation de la création, pour qu'elle soit reconnue comme telle. Le conseil des professionnels du tourisme est une pierre angulaire sur laquelle repose l'essor de l'activité. Les produits créatifs ne peuvent pas durablement s'épanouir s'ils ne sont pas enracinés. La créativité doit donc aussi infuser la mise à dispo- sition des produits. En prenant le cas de l'île Maurice, Julie Peghini (2012, p. 239) se soucie de constater que les acteurs du développement touristique sont étrangers au monde de la culture et elle pense que les profits engendrés ne sont pas réinjectés au bénéfice des industries créatives. Pour le moment, en Europe, la technologie est surtout considérée localement comme un mode de déchargement de tâches pour le personnel : elle met à disposition du client de l'information brute, pour laquelle l'agent d'accueil n'apporte pas de réelle plus-value. Dans ce cas, l'agent est un intermédiaire remplacé par des écrans (que, de toute façon, il lit au client quand celui-ci vient le voir). Il peut dès lors se consacrer plus longuement à son rôle de conseil et aider les visiteurs à trouver le produit qu'ils désirent. Il est invité à réinventer son métier, en acquérant une nouvelle dimension du territoire en développement, qui s'ajoute aux précédents atouts. Le monde du tourisme ne peut toutefois se poser uniquement comme facilitateur de cette révolution, au risque de voir - Jean Mochon le rappelle - sa légitimité remise en cause. L'ensemble du territoire doit donc être rendu réceptif aux nouveautés et à l'innovation (Mochon, 2012, p. 18).

Le comportement des e-consommateurs est révélateur des mutations actuelles. En Chine, se défiant des vendeurs et des publicités, ils s'appuient sur la recommandation des amis, devant les moteurs de recherche, le push mail et les comparateurs. $90 \%$ des pratiquants 
assidus s'informent sur les marques via les réseaux sociaux; $10 \%$ (contre $5 \%$ en France, selon l'étude de PricewaterhouseCoopers réalisée par sondage en 2011) déclarent avoir acheté via ces mêmes réseaux. Claude Vincent (2012) explique aussi ce phénomène par la faiblesse de la structuration des réseaux commerciaux, beaucoup plus éclatés qu'en Europe. Les structures françaises disposent donc d'un atout essentiel pour porter la valeur touristique de leur territoire. La valorisation du capital "confiance historique" représente pour les enseignes de distribution, voire les fabricants de marque, un enjeu fort pour progresser face aux pure players, car la distribution en ligne n'a pas, à quelques exceptions et pour l'instant, cassé les frontières (PricewaterhouseCoopers, 20II). Les seuls produits aisément étiquetés sont issus de la continuité du e-tourisme : site internet visible depuis son smartphone, codes barres bidimensionnels estampillés sur la communication ou sur des monuments. Le nombre d'applications n'est limité que par les magasins Android, Apple, Blackberry ou Windows, qui ont tout intérêt à montrer une taille critique imposante. Cette frénésie ne doit pas conduire à la boulimie. Parmi la centaine de milliers d'applications disponibles, et malgré l'existence du moteur de recherche, retrouver une application peut s'avérer une gageure. Paris et sa région compte ainsi des milliers d'applications. Confronté au choix ou, plus précisément, à la multitude, le visiteur est noyé par l'offre, qui ne se distingue pas suffisamment. Le produit gratuit a l'avantage d'un coût nul pour lui (à l'extrême, son téléchargement consomme du data) mais le contenu est de qualité variable. Avant de lancer l'application, le touriste ne sait pas sur quoi il va tomber. L'absence de coût monétaire va être compensée par du temps consacré au jugement. La marque - à l'instar des éditions papier des guides - rassure, mais l'application n'est pas gratuite. La marque pure player (modèle gratuit ou payant) convient à celui qui aura téléchargé une première application de ce type et n'en aura pas été déçu. Pour pallier ces difficultés, la ville de Paris a adoubé en juin 2011 une plate-forme web : les applications sont critiquées par la rédaction de Paris.fr et un jury doit attribuer un label aux meilleures d'entre elles. L'initiative porte surtout sur les applications Iphone (118 testées en février 2011), et débute pour les applications Android (21), Blackberry (10) et Symbian (10). Alors qu'une destination comme Paris a moins de chances de décourager l'impétrant de trouver une application intéressante, ailleurs la nécessité d'organiser l'intérêt s'est fait sentir. Le découragement sera plus rapide pour d'autres sites, même si le nombre d'applications a tendance à décroître avec la moindre affluence touristique. Le tourisme créatif a vocation à être agréable à vivre, en permettant l'épanouissement. Cet objectif doit être atteint avant même de prendre en main les outils technologiques, mais sans tomber dans un excès de stan- dardisation que Greg Richards (20 I0, p. I I) regrette d'ailleurs chez un tourisme culturel victime de son succès, d'où l'importance d'une bonne coordination des initiatives.

\section{Une offre en réseau ?}

Les réseaux sociaux connaissent actuellement une rationalisation de leur usage. De nouveaux acteurs encouragent la conciliation des multiples canaux, afin de réduire une occupation chronophage suite à l'explosion de leur popularité. Une réflexion de ce type est également nécessaire pour les produits touristiques. Il n'existe pour l'instant aucune réelle organisation de l'offre dans le m-tourisme. À l'instar des agences de voyage ou des agences hôtelières en ligne, une réflexion sur un (ou plusieurs) bub numérique doit être engagée, permettant d'agréger l'offre globale des acteurs touristiques, disponible depuis son smartphone (applications mais aussi Web-applications, sites internet...). La créativité passe donc aussi par un réseau, qui peut n'être qu'un simple référencement mais qui doit surtout donner envie de découvrir. À l'instar de l'accueil local, un renouvellement du mode de proposition d'outils numériques, jusqu'alors essentiellement chaotique et cacophonique, est indispensable. Pour l'instant, afin de pallier le manque, les acteurs les plus actifs, exploitant leur marque, proposent une page sur les réseaux sociaux. Les offices du tourisme sont conscients que les stratégies digitales les plus efficaces 
passent par une page Facebook (96\%), un compte Twitter (89\%), l'e-mailing (85\%), une application mobile (83\%) (Metz, 20 I I, p. 27), mais ces outils doivent être associés et s'inscrire dans une stratégie globale. Les rôles respectifs du ministère chargé $\mathrm{du}$ Tourisme, du Conseil national du tourisme, des comités régionaux et départementaux du tourisme sont sans doute à distribuer rapidement en la matière. Les auteurs du rapport sur le m-tourisme (Idate et Kanopée, 20 I I, pp. I I4I16) préconisent la création d'un minisite mobile local, formule économique et adaptée, pour capter le trafic naturel généré par les moteurs de recherche avec les mobinautes, une coordination avec les infomédiaires (Booking.com, Hotels.com, Tripadvisor...), et une anticipation sur le mobile sans contact. Selon eux, d'ici quelques années, une dizaine d'applications téléchargées à des millions d'exemplaires serviront de référence à la quasi-totalité des touristes mobinautes ; les offices de tourisme et autres organismes institutionnels sont donc, qu'ils le veuillent ou non, partie prenante d'un jeu concurrentiel avec les infomédiaires dont l'enjeu est la captation d'audience à partir de la diffusion gratuite d'informations.

L'attrait touristique peut aussi être renouvelé par de nouvelles façons de présenter le tourisme, et non plus seulement la destination. Actuellement, les applications sont surtout d'un abord jetable : elles se limitent à leur côté pratique. La société américaine Flurry constate en 2011 que seulement $15 \%$ des usagers continuent à les utiliser un mois après l'installation (Quignon, 20।I). Wipolo, grâce à une application Facebook, propose d'agréger les informations collectées par le voyageur pour qu'il puisse partager son aventure avec ses amis. Pour le fondateur de cette entreprise, faire connaître son voyage entraîne une émulation collective à voyager. Des applications pour smartphones, comme l'application Touristic'Tour, cherchent aussi à "inviter à partager ses points de visites favoris avec ses amis et sa famille ${ }^{(6) !}$. Néanmoins, nous abordons là un point discutable, à rapprocher du vieux débat sur les chemins de fer inventeurs d'intérêt touristique : la majorité ne voyage pas pour voyager, mais pour aller quelque part. La destination reste pour l'instant plus importante que le mode de transport. Mais les acteurs doivent être conscients d'une chose : la technologie ne remplace pas la destination, elle la sublime, un point sur lequel le tourisme créatif a beaucoup à apporter. Le m-tourisme est sans doute pour l'instant trop récent pour que l'on en comprenne réellement les impacts, et c'est pourquoi les auteurs du rapport sur le m-tourisme (Idate et Kanopée, 201 I, p. 119) préconisent de réfléchir d'abord sur le tourisme dans sa globalité plutôt que sur le "mobinaute" ou, selon l'univers du marketing (Metz, 20। I, p. 3), "touristonaute".

\section{Quelles limites à la créativité ?}

En règle générale, les propriétaires de smartphones n'achètent jamais d'applications, ou très rarement. La part du payant dans les téléchargements effectués sur Iphone représente $25 \%$ et seulement $10 \%$ pour les possesseurs d'Ipod Touch (Arbey et al., 20 I 0, p. 42). Selon le Journal du Geek du 30 septembre 2013, seulement $2 \%$ des applications Iphone payantes portent sur le voyage ${ }^{(7)}$. Le financement de l'innovation risque donc de se produire par l'intermédiaire des crédits territoriaux. Marc Casteignau (20/2, p. 32) craint justement que, dans ce domaine, on assiste à un écart croissant entre les sites principaux et les territoires désertés par ces investissements, petites collectivités ou territoires ruraux où le patrimoine et la culture ne sont pas des enjeux économiques et sociaux majeurs. Pour Guidéapolis (20 I2), l'année 2011 représente une maigre récolte de nouveautés dans les fonctionnalités, du fait des budgets mobile plus serrés, notamment quand deux applications (Iphone et Android) sont à développer : cela se traduit par une exigence moindre. Lorsque le marché public a été passé par le syndicat intercommunal, en 2010, pour l'application du Bassin d'Arcachon, les documents spécifiaient que les propositions attendues devaient justifier une dépense proche de 20000 euros, une somme que toutes les structures ne peuvent pas consacrer à de tels outils. Un projet de création et d'installation de borne interactive de visualisation en réalité augmentee Ray-On (dispositif équipé d'un écran et d'une caméra permettant de visionner un décor réel dans lequel ont été implantés un ou plusieurs modèles numériques), déve- 
loppée par la société On-situ, se serait élevé aux alentours de 60000 euros en 2012 (Eck, 20l2, p. 5). Certains investissements sont clairement réservés aux très grandes structures : le coût du projet du parcours pédestre en réalité augmentée “Imayana” s'est élevé à 3,8 millions d'euros (Plantier et Barbier, 2013, p. 35). Pour rentabiliser de telles opérations, il faut absolument trouver une interopérabilité.

Or, ce qui apporte le plus d'innovations dans le tourisme, c'est souvent l'action collaborative entre employés, consommateurs, organisations, et non une réduction à des actes individuels (Huijbens et al., 2009). Les structures touristiques peuvent ainsi détourner des outils technologiques existants pour leur propre usage. De part son instantanéité et la clarté possible de l'information délivrée, Twitter représente un moyen d'information intéressant pour le touriste qui, une fois sur place, souhaiterait savoir où manger, que faire le soir ou encore où pratiquer telle activité sportive. L'outil devient d'autant plus pertinent lorsque la destination crée une communauté de followers qui peuvent eux aussi répondre aux questions des visiteurs, se substituant ainsi à l'office de tourisme, par exemple pendant ses heures de fermeture. L'expérience de Travel Portland sur Twitter a débuté au cours de l'année 2009. Un "twisitor centre" (expression venant de la contraction de visitor centre et de twitter) a été conçu : la destination définit un hashtag, en l'occurrence \#inPDX, que le mobi- naute inclut dans sa question, ce qui permet à la destination de visualiser tous les tweets la concernant et d'y répondre dès que possible (Arbey et al., 20।0, Pp. 48-50). Il existe deux comptes Twitter pour Travel Portland : le compte Travel Portland (@TravelPortland), pour des informations génériques et des renseignements, est actif (6 995 tweets et 39 598 abonnés, le 5 octobre 2013), tandis que le compte de Travel Portland PR (@PRinPDX), ouvert le 3 septembre 2009 et plus axé sur des histoires autour de Portland, fonctionne modestement (271 tweets et 352 abonnés, le 5 octobre 2013), avec une mise en sommeil entre juillet 2010 et avril 2012. Depuis, l'entreprise GoSeeTell Network Inc., à l'origine avec Travel Portland du premier centre, a créé le site Twisitor Center [www.twisitorcenter.com], recensant toutes les structures touristiques dans le monde ayant un compte Twitter, soit 860 comptes le 5 octobre 2013. Pour faire vivre la communauté, répondre promptement à toute question, relayer les idées, il importe néanmoins qu'une personne s'y consacre spécifiquement. En suivant cette stratégie, les structures touristiques doivent se comporter, en partie, comme des entreprises de gestion de risques très réactives, mélangeant communication verticale et communication horizontale. Il faut choyer le touriste, non pas comme un être sans responsabilité mais comme une personne au potentiel à affirmer (Richards, 20I I, p. 1243 ; Gravari-Barbas, 20।3, pp. 74-76). Les circuits d'interprétation patrimoniaux fonctionnant par QR codes pourraient permettre le visionnage, après vérification, de vidéos réalisées par les habitants ou les touristes eux-mêmes ; et les applications pour smartphone pourraient grossir grâce à la multitude d'informations glanées par les curieux. Ces enjeux risquent de bousculer bien des structures touristiques, mais aussi les professionnels du secteur.

\section{Conclusion}

Plusieurs freins conditionnent l'appropriation des nouvelles technologies par les agents touristiques et, en partie, par les touristes : le contrôle de l'image reste la prérogative des structures, qui craignent, telles des tâches indélébiles, les débordements à ce sujet ; les investissements restent lourds car il ne suffit pas de s'équiper, il faut aussi promouvoir et accompagner ; conséquence d'une innovation récente, les retombées effectives demeurent mal connues. Finalement, que ce soit en matière de technologie ou de tourisme créatif, les mêmes craintes subsistent. Dans ces conditions, le tourisme créatif risque de peiner à s'épanouir dans les nouvelles technologies. Greg Richards (2010, p. 15) est d'ailleurs bien conscient du manque de recul concernant les pratiques, pour lesquelles il faut encore déterminer les différents modèles de développement selon les caractéristiques de la destination et des co-créations entre agents touristiques et consommateurs. Le rapport Nogué (2013, pp. 27-28) promeut un marketing territorial plus développé, des contrats de destina- 


\section{DOSSIER • TOURISME CRÉATIF}

tion pour fédérer les structures et une ingénierie touristique à organiser sous l'égide d'Atout France.

Actuellement, comme le pensent certains observateurs du secteur, tel Guidéapolis (20/2), la production technologique touristique entre dans une phase de maturité. Le secteur se trouve à la croisée des chemins : continue-t-il d'être majoritairement soutenu par la puissance publique, en tout cas pour la France, quitte à rester dans ce giron sans exploiter les connaissances des touristes ?; cherche-t-il à agréger les contenus épars développés par les visiteurs, quitte à parasiter son message et à mettre en danger la confiance qu'on lui accorde ? ; doit-il coordonner mission publique et création privée, quitte à apparaître un acteur de dossiers qui progressivement s'éloigne du terrain ? Le champ des possibles reste ouvert. Encore faut-il qu'il soit investi. Le mouvement de l'open data, timoré dans le secteur touristique, pourrait à nouveau le bousculer $^{(8)}$. Nouvelles technologies et tourisme créatif, parfois associés, sont une nouvelle porte d'accès au territoire qu'il n'est décidément pas facile psychologiquement de franchir.

\section{Notes}

(2) [www.barbatre.fr/tourisme/animations/b alisages].

(3) Je remercie Christophe Renaudineau et Francky Trichet, d'Iréalité, entité coréalisatrice de l'opération Balis'Âges, pour l'ensemble de ces informations statistiques. Je précise que j'ai été chargé de mission lors de cette opération.

(4) [www.globalgreeternetwork.info].

(5) Consultation du dossier de presse sur le site des greeters de Nantes [www.greeters-nantes.com/presse.pdf]

(6) Dépliant promotionnel de l'application Touristic'Tour, 2012. Je précise que j'ai été consulté pour l'architecture de cette application par la société Mobile

Développement.

(7) "Pour I, I 3 million de dollars, offrezvous toutes les applications payantes de l'App Store"

[www.journaldugeek.com/20 I3/09/30/pour - I 13 -millions-de-dollars-offrez-vous-toutes- les-applications-payantes-de-lapp-store]. Les informations de cet article proviennent de l'article d'Eric Monti, "Million dollar expense: buying out the App Store" [http://gigaom.com/2013/09/29/million-dollar-expense-buying-out-the-app-store], paru le 29 septembre 20 3. Je remercie le journaliste Gregori Pujol pour cette précision. (8) L'équipe de rédaction d'Espaces tourisme et loisirs, "Open data et tourisme", note de synthèse, août 2013 ; Sabine Blanc, "Open data et tourisme : un potentiel qui reste à transformer", lagazette.fr, 23 septembre 2012 [www.lagazettedescommunes. com/195963/open-data-et-tourisme-unpotentiel-qui-reste-a-transformer].

\section{Références bibliographiques}

Maxime Aмıот, "La géolocalisation veut entrer dans les centres commerciaux", Les Échos, 21 février 2012.

Emeline Arbey, Cécile Austin, Loïc CoIssin, Charline Feral, Carolyn GILleSPIE et Marie LANGUILle, Un enjeu pour l'OT de demain : comment adapter les services accueil et information aux nouvelles attentes des touristes?, mémoire de master 2 , Institut d'aménagement, de tourisme et d'urbanisme-Bordeaux III, 2010

François BostnaVARON, "Le guide touristique papier fait de la résistance", Lemonde.fr, 23 juillet 2012.

Marc Casteignau, "Nouvelles technologies, nouvelle médiation patrimoniale", Espaces tourisme et loisirs, n³00, février 2012. Jean-Marc CieUtAT, Quelques applications de la réalité augmentée nouveaux modes de traitement de l'information et de la communication. Effets sur la perception, la cognition et l'action, mémoire pour l'obtention du titre d'habilitation à diriger des recherches en informatique, Université Toulouse III-Paul Sabatier, 2013. 
Sophie DESHAYES, "Audioguides et musées",

La Lettre de l'Ocim, no 79, 2002.

Nicolas EcK, Conception et réalisation d'un prototype de borne interactive destinée à la visualisation en réalité augmentée de deux modèles numériques des fortifications de Nancy Renaissance, projet de fin d'études de $5^{\circ}$ année à l'Insa de Strasbourg, 2012.

Jessica Fèvres-DE BIDERAN, "La réalité augmentée, une nouvelle vision du patrimoine", Espaces, tourisme et loisirs, n 314 , septembre-octobre 2013.

\section{Nehla GhoualeL, Jean-Marc Cieutat et Jean-Pierre JeSSEL,}

"MARTS : un système mobile de réalité augmentée dédié au tourisme", Mobilité et Ubiquité 2011 , Actes des 7e Journées francophones, Ubimob'।I, Toulouse, 6-8 juin, 201 I.

Maria Gravari-BaRBAS, Aménager la ville par la culture et le tourisme, coll. "Ville-Aménagement", Le Moniteur, 2013.

GuIDÉAPOLIS, "Classement des applications iPhone des offices de tourisme 2012 ", juin 2012 [http://www.guideapolis.com/fr/blog/classement-des-applications-iphone-des-offices-de-tourisme-20 /2/].

\section{Edward Hákon HuijBens, Anne-Mette Hjalanger, Peter Björk,}

Sara NORDIN et Arvid FLAGESTAD, "The role of innovations

systems", dans Jovo Ateljevic et Stephen J. Page (dir.),

Tourism and Entrepreneurship: International Perspectives, ButterworthHeinemann, 2009.

IDATE et KanopéE, Prospective du m-tourisme,

rapport du Pipame, novembre 201 I.

Géraldine METZ, Tourisme et réseaux sociaux, dossier technique $n^{\circ} 5$, master 2 Gestion et droit de l'économie numérique, Université de Strasbourg, 201 I.

Jean MOCHON, "L'innovation, moteur du renouvellement de l'information et de la médiation", Espaces tourisme et loisirs, n 300,

février 2012.

François NoguÉ, Le Tourisme, "filière d'avenir" : développer l'emploi dans le tourisme, rapport du ministère de l'Artisanat, du Commerce et du Tourisme et du ministère du Travail, de l'Emploi, de la Formation professionnelle et du Dialogue social, novembre 2013. Claude Origet du Cluzeau et Patrick Vicériat, Le Tourisme des années 2020 : des clés pour agir, La Documentation française, 2009.
Julie PEGHINI, "Les industries créatives à l'île Maurice : vers un renouvellement des initiatives publiques et privées ?", dans Philippe BovQuiluion (dir.), Creative Economy, Creative Industries : des notions à traduire, Presses universitaires de Vincennes, 2012.

Donna Pendergast, "Getting to know the Y generation", dans Pierre BenCKendorfF, Gianna MosCardo et Donna Pendergast (dir.), Tourism and Generation Y, CAB International, 2010.

Bruno PLANTIER et Thierry BARbIeR, '“Imayana' fait vivre BordeauX au XVIII' siècle", Espaces tourisme et loisirs, $n^{\circ} 3 \mid 4$, septembre-octobre 2013.

PrICEWATERHOUSECOOPERS, Web-acheteurs : comportement et motivations, France et international, rapport d'étude, décembre 201 I. Catherine QUIGNON, "Applications smartphones - Gare à l'effet 'Kleenex"', LenouvelEconomiste.fr, 23 mars 201 I.

Greg RICHARDS, "Tourism development trajectories - from culture to creativity?", Revista Encontros Cientificos - Tourism \& Management Studies, $n^{\circ}$ 6, 2010.

Greg RICHARDS, "Creativity and tourism. The state of the art", Annals of Tourism Research, vol. 38, $n^{\circ} 4,2011$.

Éric SeULliet, "Comment la technologie nous rend plus créatifs", communication à la $2^{e}$ conférence internationale organisée par l'Adcep : Le Tourisme à l'heure du touriste créatif, Paris, 6-7 décembre, 2012 [www.tourisme-creatif.org/?page_id=508].

Florence TousSAINT, "Approche des industries créatives : origine et définition de la notion", dans Philippe BouQuILıION (dir.), Creative Economy, Creative Industries : des notions à traduire, Presses universitaires de Vincennes, 2012.

Jean-Didier URBAIN, L'Idiot du voyage : histoires de touristes, Payot, 2002 (Ire éd. 199|).

Jean-Didier URBAIN, Le voyage était presque parfait : essai sur les voyages ratés, Payot, 2008.

Claude VINCENT, "Les Chinois accros au e-shopping", Enjeux Les Échos, n²87, février 2012.

Dan Wang, Sangwon Park et Daniel R. Fesenmaier, "An examination of information services and smartphone applications", $16^{\text {th }}$ Graduate Students Research Conference in Hospitality and Tourism, UMassAmherst, 201।. 